\title{
Caracterización de la materia prima para la elaboración de un material de construcción utilizando diatomita y Residuos de Construcción y Demolición (RCD) Characterization of the raw material for the elaboration of a construction material using diatomite and Construction and Demolition Residues (RCD)
}

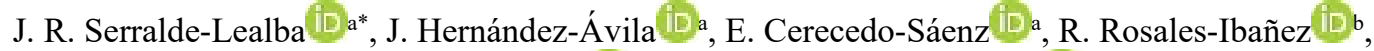 \\ E. Salinas-Rodríguez a , F. R. Barrientos-Hernández \\ a Área Académica de Ciencias de la Tierra y Materiales, Universidad Autónoma del Estado de Hidalgo, 42184, Mineral de la Reforma, Hidalgo, México. \\ ${ }^{\mathrm{b}}$ Facultad de Estudios Superiores Iztacala, Universidad Nacional Autónoma de México, 54090, Tlalnepantla de Baz, México.
}

\begin{abstract}
Resumen
Se caracterizó los Residuos de Construcción y Demolición (RCD), para determinar un uso alternativo de este material en la elaboración de bloques de pumicita. A partir de la utilización de RCD y diatomita para mejorar sus propiedades mecánicas. Los resultados obtenidos señalaron que estos RCD están compuestos principalmente de aluminio, silicatos y carbonatos, como fases mayoritarias. Se utilizó para producir materiales de construcción (bloques) con propiedades de resistencia bastante similares o incluso superiores a los presentados por materiales comerciales. Las propiedades más importantes encontradas con el uso de diatomita y de RCD en la incorporación de bloques de pumicita están relacionadas con valores como resistencia a la compresión, mostrando resultados que varían de 22 a 24.4 $\mathrm{kg} / \mathrm{cm}^{2}$, superiores a los comerciales que presentan una resistencia de $20 \mathrm{~kg} / \mathrm{cm}^{2}$, teniendo un aumento del $20 \%$, aunado a la disminución en la cantidad de cemento al usar diatomita en concretos, ahorrándose 2 bultos ( $100 \mathrm{Kg}$ ) por cada 20 bultos ( 1 Ton) de cemento y cuya mejora genera aumentos a la resistencia a edades tempranas, llegando al $90 \%$ de resistencia a la compresión en 10 días.
\end{abstract}

Palabras Clave:

Reciclaje, Sustentable, Bloque, Puzolana, Diatomita.

\begin{abstract}
Construction and Demolition Residues (RCD) were characterized to determine an alternative use of this material in the elaboration of pumicite blocks from the use of RCD and diatomite to improve their mechanical properties. The results obtained by DRX and SEMEDS and M. Optic, indicated that these RCD are mainly composed of aluminium, silicates and carbonates, as the majority phases. It was used to produce building materials (blocks) with some strength properties quite similar or even superior to those exhibited by commercial materials. The most important properties found with the use of diatomite and RCD in the incorporation of pumicite blocks are related to values such as compressive strength that show results that vary from 22 to $24.4 \mathrm{~kg} / \mathrm{cm}^{2}$, higher than the commercial ones that present a resistance of $20 \mathrm{~kg} / \mathrm{cm}^{2}$, having an increase of $20 \%$, coupled with the decrease in the amount of cement when using diatomite in concrete, saving 2 packages $(100 \mathrm{Kg}$ ) for every 20 packages ( $1 \mathrm{Ton})$ of cement and whose improvement generates increases resistance to early ages, reaching $90 \%$ compressive strength in 10 days.
\end{abstract}

Keywords:

Recycling, Sustainable, Block, Pozzolana, Diatomite.

\section{Introducción}

El reciclaje ha adquirido mayor impacto, principalmente en el ámbito de la construcción ya que esta industria contiene bastantes elementos cuya gestión no es llevada a cabo de la forma correcta como los Residuos de Construcción y Demolición (RCD); y producen gran huella de carbono, entre ellos se encuentra el cemento, cuyo impacto ambiental genera un 7\% de las emisiones totales de $\mathrm{CO}_{2}$ en el mundo (Oh et al, 2014). La sostenibilidad en la industria de la construcción es inevitable y no solo significa que se debe reducir la huella de carbono, sino que también debe contribuir a conservar los recursos naturales que son cruciales para el crecimiento continuo (Akhtar \& Sarmah, 2018). Por lo que, al crear materiales de construcción que sean sustentables y con

*Autor para la correspondencia: jurusele@gmail.com

Correo electrónico: jurusele@gmail.com (Juan Rubén. Serralde-Lealba), herjuan@uaeh.edu.mx (Juan Hernández-Ávila), mardenjazz@yahoo.com.mx (Eduardo Cerecedo-Sáenz), dr.raul.rosales@gmail.com (Raúl Rosales-Ibañez), salinasr@uaeh.edu.mx (Eleazar Salinas-Rodríguez), frbh68@hotmail.com (F.Raúl Barrientos-Hernández) 
mejores características que los existentes, es una forma en la cual se puede reducir el impacto ambiental generado también por los Residuos de Construcción y Demolición.

Cada año se producen grandes cantidades de estos desechos, los cuales son considerados inertes, no peligrosos y poseen alta susceptibilidad de ser aprovechados mediante transformación y reincorporación como materia prima de agregados en la fabricación de nuevos productos. En su mayoría, los agregados de concreto reciclado están compuestos de una mezcla de pasta de mortero endurecida adherida. Una gran parte de ellos se podría reutilizar en la fabricación de nuevos materiales de construcción para disminuir las cantidades de desechos cuyo destino final son vertederos clandestinos como barrancas o terrenos cuyo uso se podría aprovechar para realizar actividades productivas y así preservar los recursos naturales (Serralde et al, 2015). El concepto de reciclaje de los RCD se ha estado abordando en diversos trabajos de investigación anteriores, sin embargo, son investigaciones a nivel local, por lo que en México aún no se tiene un control total de la disposición final de estos escombros.

Los bloques de pumicita son utilizados en la industria de la construcción como elementos perimetrales y divisorios en edificaciones, creando muros sólidos cuyas dimensiones son variables. Se consideran bloques de tepojal-cemento, donde su uso recae en un material no estructural en base a la normativa mexicana NMX-C-441-ONNCCE-2013. Una de las propiedades mecánicas más importantes de los bloques es la resistencia a la compresión. Las propiedades mecánicas pueden mejorarse con materiales alternativos utilizados para el reemplazo parcial del cemento Portland. Uno de estos materiales es la diatomita, que es una roca silícica de tipo fósil que se formó por la acumulación sedimentaria de capas silíceas de algas, compuesta de esqueletos opalinos de sílice amorfa y tiene una fórmula química de $\mathrm{SiO}_{2}-$ $\mathrm{nH}_{2} \mathrm{O}$ (Mete, 1988). Este tipo de $\mathrm{SiO}_{2}$ puede reaccionar con $\mathrm{Ca}$ $(\mathrm{OH})_{2}$ y producir hidratos de silicato de calcio $(\mathrm{CSH})$, que son responsables del desarrollo de la resistencia. Se caracteriza por ser una puzolana natural $\mathrm{y}$ ha sido utilizada en diversas investigaciones como aditivo puzolánico para cemento portland (Aydin \& Gül, 2007). La reacción puzolánica de la diatomita conduce a la formación de mayores cantidades de CSH, especialmente a la edad de 28 días (Kastis, 2006). El uso de rocas de diatomita como aditivos de cemento tiene inconvenientes tales como una mayor demanda de agua, pero la resistencia a la compresión de los cementos producidos en laboratorio exhibe valores más altos que los del cemento Portland de referencia (Stamatakis et al 2003).

Por lo tanto, el objetivo de este trabajo es caracterizar la mineralogía y la composición química de los agregados reciclados de concreto demolido de la Ciudad de México, de diatomita tanto natural como calcinada y pumucita, para demostrar la posibilidad de usar este material reciclado, y eventualmente generar nuevas oportunidades de mercado (Cardoso et al, 2016).

\section{Procedimiento experimental}

\subsection{Materiales}

Los agregados reciclados se obtuvieron de la planta de Concretos Reciclados S.A. de C.V, muestreándolo directamente del procesado montículos (1/4 "de grano a arena de grano fino y grava de 1") obtenidos por la trituración de estos en una unidad el reciclaje móvil McCloskey, modelo I44R (McCloskey International, Peterborough, ON, Canadá); por su parte la pumicita fue muestreado en el municipio de Tulancingo y la diatomita en la comunidad de "Loma Larga" en el municipio de "Acatlán", y el cemento portland compuesto se obtuvieron de una casa de materiales en Pachuca de Soto, todo estos en el estado de Hidalgo, México.

\subsection{Métodos}

\subsubsection{Caracterización Granulométrica}

Para determinar el tamaño de los agregados recicladas, se secaron muestras de $10 \mathrm{~kg}$ para agregados gruesos y $1 \mathrm{~kg}$ para agregados finos (LS-602, 2001), a $100{ }^{\circ} \mathrm{C}$ hasta un peso constante antes del tamizado. La distribución del tamaño se analizó de acuerdo con la norma NMX C-077-ONNCCE-1997 (EN 933-1: 1997), que describe análisis de tamices y métodos de prueba para agregados de concreto.

\subsubsection{Caracterización mineralógica y petrográfica}

Para la caracterización mineralógica y petrográfica de la muestra, se preparó una sección delgada y se examinó utilizando microscopía óptica polarizada transmitida en un microscopio Olympus BX41

\subsubsection{Calcinación de diatomita}

Las muestras de diatomita fueron molidas en un mortero, se realizó la calcinación a dos muestras; una utilizando fundente de hidróxido de sodio $(\mathrm{NaOH})$ al $7 \%$; y la otra sin fundente. La calcinación de las muestras de diatomita se realizó utilizando un horno marca Lindberg-ISB en atmósfera de aire ubicado en la (UAEH). El proceso se llevó acabo a $1000^{\circ} \mathrm{C}$ a una velocidad de calentamiento de $10{ }^{\circ} \mathrm{C} / \mathrm{min}$ y una parada a $1000{ }^{\circ} \mathrm{C}$ durante 1 hora, enfriándose a temperatura ambiente (Hernández et al, 2017), (Borgel, 2007).

\subsubsection{Caracterización química y mineralógica}

Para las técnicas de caracterización, fue necesario preparar la muestra a tamaños de partícula inferiores a $75 \mu \mathrm{m}$, y secar a 100 ${ }^{\circ} \mathrm{C}$ durante $6 \mathrm{~h}$ en un horno de laboratorio con un controlador digital. posteriormente se caracterizaron por los siguientes métodos de análisis: difracción de rayos $\mathrm{X}$ realizada con un difractómetro INEL modelo Equinox 2000 ubicado en Universidad Autónoma del Estado de Hidalgo (UAEH), México. La base de datos MATCH fue usado para indexar los espectros. La cuantificación de los elementos contenidos en las muestras fue realizada por espectrofotometría de plasma inducción acoplada usando un modelo 2100 de Perkin Elmer (ubicado en UAEH). La morfología de las muestras se obtuvo utilizando un microscopio electrónico de barrido, modelo JSM-IT300 (ubicado en UAEH) con un voltaje de $30 \mathrm{keV}$ y equipado con un espectrómetro de dispersión de energía de Detector de rayos X EDS, marca OXFORD. El análisis químico de fluorescencia de rayos X (XRF) se realizó mediante un dispositivo portátil BRUKER Espectrómetro XRF, modelo S1 TITAN (ubicado en UNAM).

\subsubsection{Fabricación de bloques de pumicita}

La elaboración de los bloques se llevó a cabo en 2 etapas con el fin de determinar el que mejores propiedades mecánicas presente. Primero se realizó la sustitución de cemento con diatomita en porcentajes de 5,10 y $15 \%$. En la segunda etapa se incorporaron los agregados reciclados en el bloque de pumicita en porcentajes de 10, 20 y $30 \%$. Los bloques utilizados en las primeras pruebas se agruparon como referencia, diatomita natural, 
diatomita calcinada y diatomita calcinada con fundente. El cemento de referencia se obtuvo en base a dosificaciones de diversas bloqueras y se llama R. Los bloques de pumicita mezclados con diatomita natural se obtuvieron reemplazando el cemento con diatomita natural en varias proporciones $(5 \%, 10 \%$ y $15 \%$ ) en peso y manteniendo la pumicita. Estas muestras fueron llamados $5 \mathrm{DN}, 10 \mathrm{DN}$ y $15 \mathrm{DN}$, respectivamente. De manera similar, las muestras de block mezclado con diatomita calcinada sin fundente (DC) se obtuvieron reemplazando el cemento con diatomita calcinada en varias proporciones $(5 \%, 10 \%$ y $15 \%)$ en peso y manteniendo la pumicita. Por último, las muestras de block mezclado con diatomita calcinada con fundente se obtuvieron reemplazando el cemento con diatomita calcinada con fundente $(\mathrm{NaOH})$ en varias proporciones $(5 \%, 10 \%$ y $15 \%)$ en peso y manteniendo la pumicita. Estas muestras fueron llamadas 5DCF, 10DCF y 15DCF, respectivamente. Los bloques utilizados para las segundas pruebas se obtuvieron mezclando la pumicita con los agregados reciclados en porcentajes de 10,20 y $30 \%$, manteniendo constante la cantidad de cemento y diatomita.

\subsubsection{Propiedades mecánicas}

Para las pruebas de resistencia a la compresión, se realizaron bloques de pumicita con un tamaño de $60 \times 200 \times 50 \mathrm{~mm}$. Se colocaba la mezcla y se comprimía con un pisón, una vez enrasados y comprimidos, se desmoldearon y se dejaron secar a temperatura ambiente durante 7,14 y 28 días para realizarle las pruebas de resistencia a la compresión. Las pruebas se realizaron en base a la normativa norma NMX-C-036-ONNCCE-2013 utilizando una prensa ELVEC.

\section{Resultados y discusión}

\subsection{Caracterización granulométrica}

Para determinar el tamaño del agregado reciclado y la curva granulométrica, se pesaron muestras de $5 \mathrm{~kg}$ para agregados gruesos. La distribución del tamaño se analizó de acuerdo con la norma NMX C-111-ONNCCE-2017 "Industria de la construcción - Agregados hidráulicos de concreto - Especificaciones y métodos de prueba", que describe análisis de tamices y métodos de prueba para agregados de concreto.

Tabla 1. Composición química promedio de Agregado Reciclado, diatomita y pumicita.
En la Figura 1, se aprecia que el final de la curva de los agregados reciclados está fuera del límite granulométrico ya que generan una mayor cantidad de finos en el momento de ser tamizados por la presencia de mortero recubriendo los agregados (Cáceres, 2015), por lo que el agregado reciclado presenta un tamaño nominal máximo de 15 a $20 \mathrm{~mm}$, en comparación con el tamaño promedio del agregado de piedra pómez $(10 \mathrm{~mm})$, por lo cual fue necesaria la trituración secundaria para uniformar los tamaños para poder utilizarlos como materia prima en la elaboración de los bloques dando como resultado un tamaño de partícula entre 7.5 y $12 \mathrm{~mm}$.

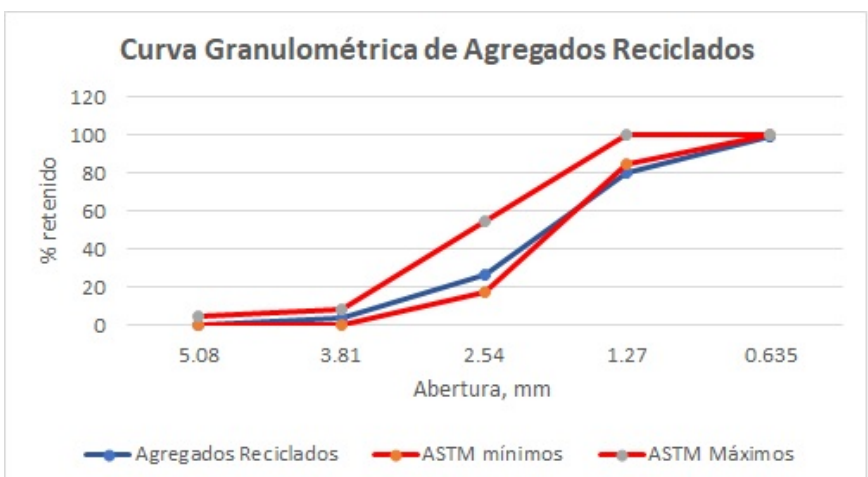

Figura 1. Distribución granulométrica de la grava reciclada: las líneas punteadas indican los límites granulométricos según la norma NMX C-111-ONNCCE-2017.

\subsection{Análisis químico}

A partir de los resultados del análisis químico por ICP, FXR Y MEB-EDS los principales elementos presentes en los residuos de los agregados pétreos son $\mathrm{Si}, \mathrm{Al}, \mathrm{K}, \mathrm{Na}, \mathrm{Ca}, \mathrm{Fe}, \mathrm{y} \mathrm{Mg}$. Mientras que para la diatomita natural se presentan contenidos mayoritarios de $\mathrm{Si}, \mathrm{Al}, \mathrm{K}, \mathrm{Mg}, \mathrm{Ca}, \mathrm{Fe}$ y elementos minoritarios como el $\mathrm{Na}$ y el Ti y para la pumicita tenemos contenidos mayoritarios de $\mathrm{Si}$, $\mathrm{Al}, \mathrm{K}, \mathrm{Mg}, \mathrm{Ca}, \mathrm{Fe}$ y como elemento minoritario el Ti, como se puede apreciar en la tabla 1 .

\begin{tabular}{|c|c|c|c|c|c|c|c|c|c|}
\hline \multirow{3}{*}{$\begin{array}{l}\text { Elemento/ } \\
\text { compuesto }\end{array}$} & \multicolumn{9}{|c|}{$\%$ Peso } \\
\hline & \multicolumn{3}{|c|}{ Arena reciclada } & \multicolumn{3}{|c|}{ Diatomita } & \multicolumn{3}{|c|}{ Pumicita } \\
\hline & ICP & FRX & SEM-EDS & ICP & FRX & SEM-EDS & ICP & FRX & SEM-EDS \\
\hline $\mathrm{Na}_{2} \mathrm{O}$ & 5.30 & 5.31 & 5.21 & 0.61 & 0.923 & 0.08 & 1.66 & 1.62 & 1.58 \\
\hline $\mathrm{MgO}$ & 4.30 & 4.32 & 4.38 & 1.79 & 1.87 & 1.87 & 0.0 & 0.0 & 0.87 \\
\hline $\mathrm{Al}_{2} \mathrm{O}_{3}$ & 8.42 & 8.44 & 9.25 & 11.63 & 7.77 & 7.77 & 14.9 & 15.54 & 15.17 \\
\hline $\mathrm{SiO}_{2}$ & 48.37 & 49.41 & 50.76 & 76.00 & 77.36 & 77.14 & 73.11 & 72.98 & 72.14 \\
\hline $\mathrm{K}_{2} \mathrm{O}$ & 1.12 & 1.14 & 1.17 & 2.41 & 1.2 & 1.12 & 5.12 & 5.01 & 5.11 \\
\hline $\mathrm{CaO}$ & 26.53 & 19.11 & 15.76 & 0.85 & 1.04 & 1.04 & 1.25 & 1.38 & 1.24 \\
\hline $\mathrm{TiO}_{2}$ & 0.57 & 0.55 & 0.56 & 0.50 & 0.348 & 0.23 & 0.33 & 0.29 & 0.23 \\
\hline $\mathrm{FeO}$ & 4.57 & 3.99 & 4.59 & 1.95 & 2.35 & 2.34 & 3.63 & 3.18 & 3.34 \\
\hline
\end{tabular}


En la Tabla 2 se muestran los resultados del análisis químico de la diatomita tratada; donde se observa que la composición química de la diatomita natural fue modificada después de la calcinación, debido a que se incrementó el contenido de sílice, se redujo el contenido de Fe y humedad, se transformaron los carbonatos, se eliminó la materia orgánica y el Al permaneció sin un cambio aparente, por consiguiente las propiedades físicas y químicas de la diatomita fueron mejoradas para su uso industrial (según la norma ASTM D 604-81).

Tabla 2. Composición química de la diatomita calcinada y calcinada con fundente.

\begin{tabular}{ccc}
\hline $\begin{array}{c}\text { Elemento/ } \\
\text { Compuesto }\end{array}$ & $\begin{array}{c}\text { Diatomita } \\
\text { calcinada sin } \\
\text { fundente }\end{array}$ & $\begin{array}{c}\text { Diatomita } \\
\text { calcinada con } \\
\text { fundente }\end{array}$ \\
\hline $\mathrm{SiO}_{2}$ & 81.50 & 82.00 \\
$\mathrm{Al}_{2} \mathrm{O}_{3}$ & 12.00 & 11.15 \\
$\mathrm{~K}_{2} \mathrm{O}$ & 2.10 & 1.96 \\
$\mathrm{Na}_{2} \mathrm{O}$ & 0.45 & 2.82 \\
$\mathrm{CaO}$ & 0.10 & 0.13 \\
$\mathrm{FeO}$ & 1.44 & 1.44 \\
$\mathrm{MgO}$ & 0.48 & 0.48 \\
$\mathrm{TiO}_{2}$ & 0 & 0 \\
\hline
\end{tabular}

\subsection{Composición mineralógica}

La Figura 2 muestra los resultados de difracción de rayos $\mathrm{X}$ de los agregados reciclados, donde se pueden observar picos anchos de fases que posiblemente representan tamaño de cristal pequeño, y traslape de reflexiones, típico de las mezclas multifase. La identificación de fases se realizó mediante el software Match, determinándose la presencia de diferentes cantidades de fases cristalinas que corresponden a los agregados originales y a la pasta de cemento adherida a ellos, como era de esperarse en un concreto.

Los minerales mayoritarios de las muestras analizadas pertenecen a los agregados primarios cuyo espectro se observa en la Figura 2, fases que coinciden con el material de base ya que normalmente, los agregados representan alrededor del 70\% - 80\% de los componentes de los concretos (Omary et al, 2016). Así mismo, se identificaron feldespatos triclínicos (plagioclasas de $\mathrm{Na}$ y Ca), como albita, oligoclasa, andesina, además de anortoclasa que también es un feldespato, pero de $\mathrm{Na}$ y $\mathrm{K}$, y que posee una estructura triclínica al igual que las plagioclasas. Estos minerales son en realidad una solución sólida, de ahí el ensanchamiento de los picos (marcados con Fd), que corresponden a la misma reflexión, pero con una celda unitaria con diferente cantidad de átomos de $\mathrm{K}$, $\mathrm{Na}$ y $\mathrm{Ca}$, que cambia ligeramente la estructura cristalina del feldespato, evidenciando la diferencia del tamaño de los átomos (Moreno et al, 2018).

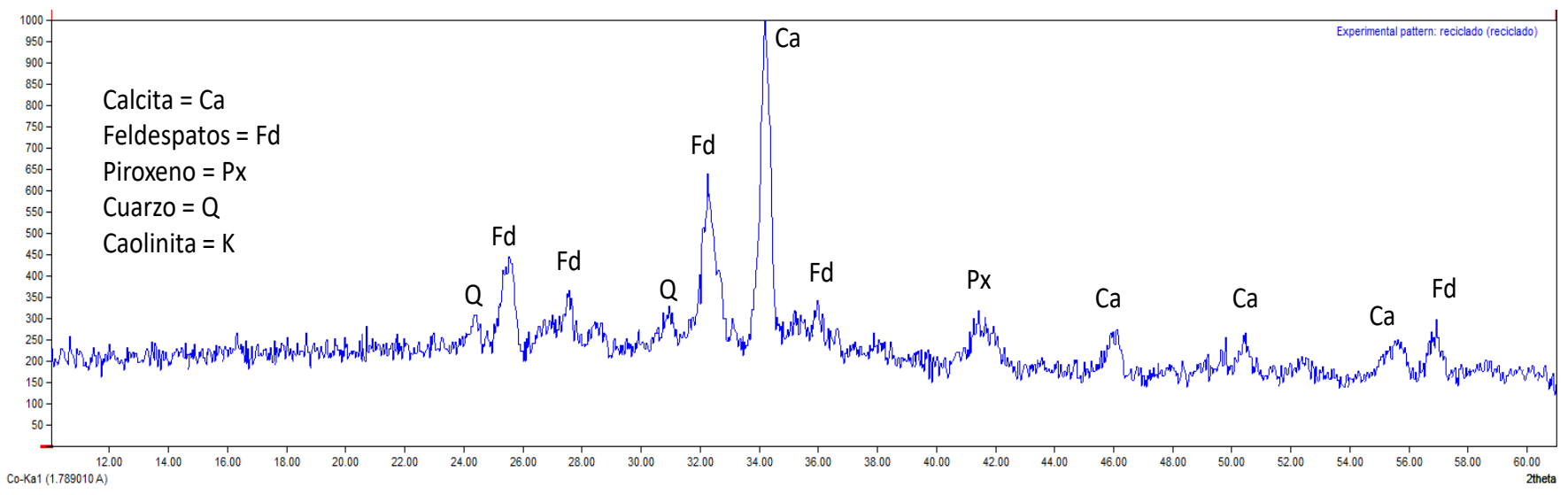

Figura 2. Patrones de difracción de rayos $\mathrm{X}$ en las muestras de arena reciclada

Entre tanto, en la Figura 3, las principales especies mineralógicas identificadas en la diatomita por difracción de Rayos X fueron cuarzos, albita, bernilita, en donde la especie mayoritaria es la sílice. En la Figura 3 también se aprecia la evolución de las fases cristalinas de la diatomita con diferentes tratamientos, en el espectro inferior de diatomita, se observa el ensanchamiento de los picos y un mayor ángulo 2 Theta de la fase de cuarzo cercano a los $27^{\circ}$ y a $43^{\circ}$ en forma gaussiana que muestra que el tamaño de los cristales es menor con respecto a los otros espectros y la cristalinidad menor.

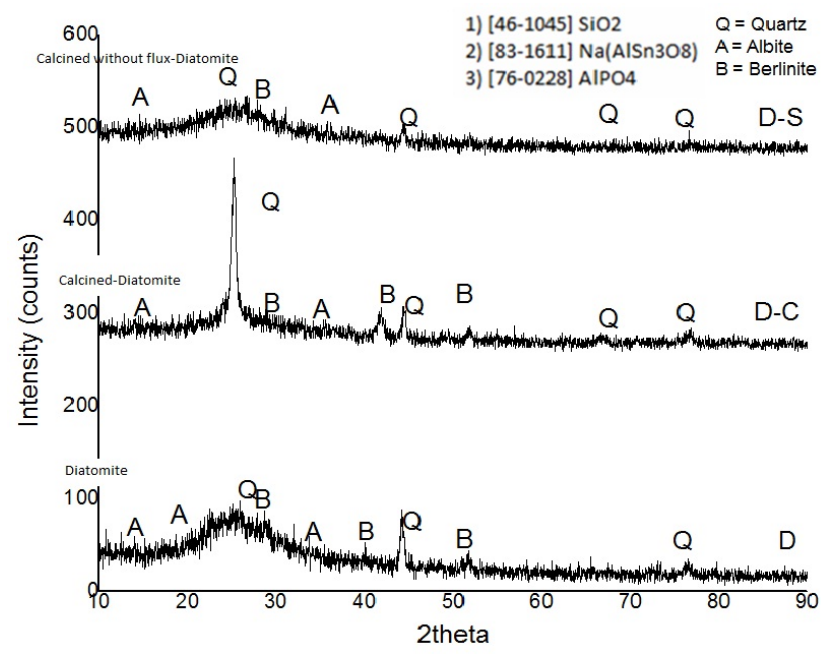

Figura 3. Espectro de la diatomita natural y calcinada con y sin fundente 
Entre tanto, en el espectro de diatomita calcinada se observa un pico muy definido y nítido cercano a $25^{\circ}$, que muestra un aumento del tamaño del cristal y los ángulos menores en 2theta muestran que creció el tamaño de cristal y la fase de berlitita a $40^{\circ}$ cristalizo. Así mismo en el espectro superior sin fundente se observa una deficiencia de berlinita donde aparece con un ángulo menor a $29^{\circ}$ y un posible crecimiento del cristal y posiblemente mayor crecimiento amorfo del espectro.

En la Figura 4, se observan especies minerales típicas de pumicita identificadas por Difracción de Rayos X, con fases minerales mayoritarias de feldespato con cristobalita muy nítido cercano a $24^{\circ}$ en 2 theta, paragonite, gmelite y berlinita

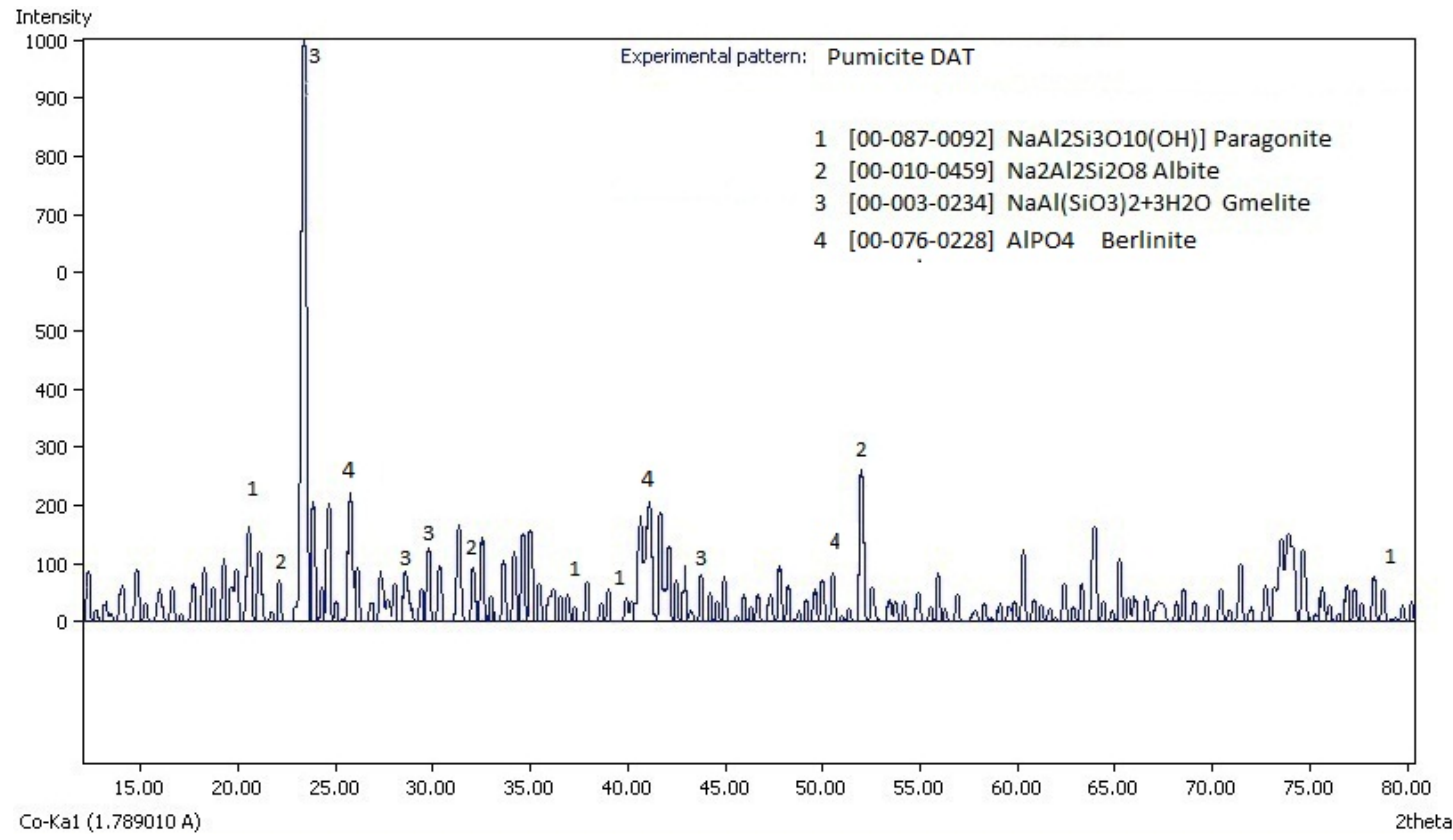

Figura 4. Espectro de Rayos X de pumicita.

\subsection{Microscopía óptica}

En las Figura 5, se muestra la petrología de la sección delgada del Agregado Reciclado donde se observa la presencia de minerales de plagioclasa, albita, cristales de augita y cuarzo.

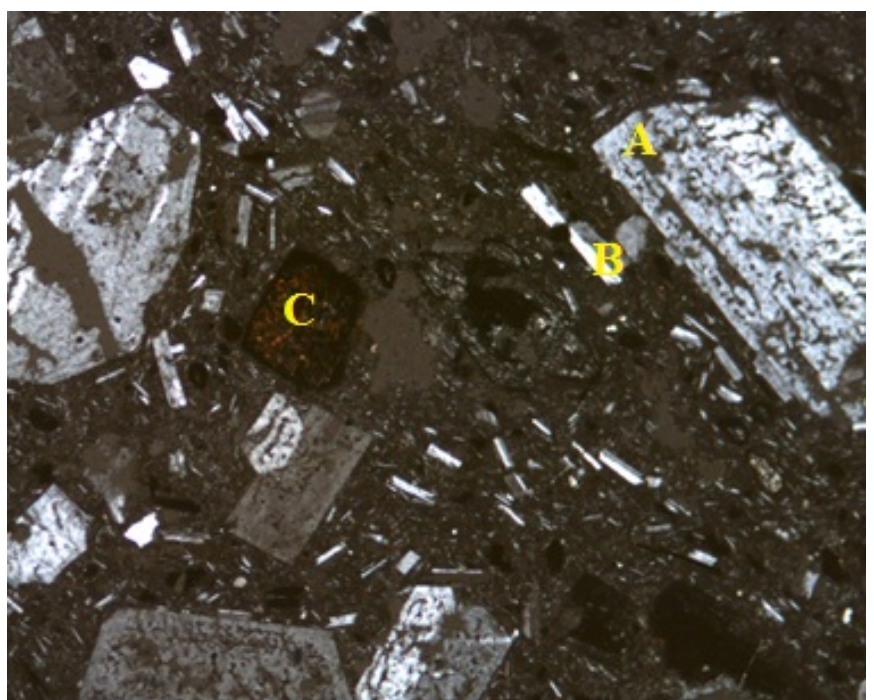

Figura 5. Imagen de microscopio de luz transmitida (nicoles cruzados, $4 \mathrm{X}$ ), mostrando cristales de plagioclasa (A) rodeados por una pasta microlítica de láminas de plagioclasa (B). Minerales opacos y un único cristal de anfíbol de augita (rojizo) (C).
En la Figura 6, se puede observar que la plagioclasa está perfectamente cristalizada (A) y muestra la concurrencia de zonaciones de origen Volcánico (B), con las características maclas polisintéticas de albita paralelas al alargamiento del cristal (C), y cristales de augita (D) y cuarzo.

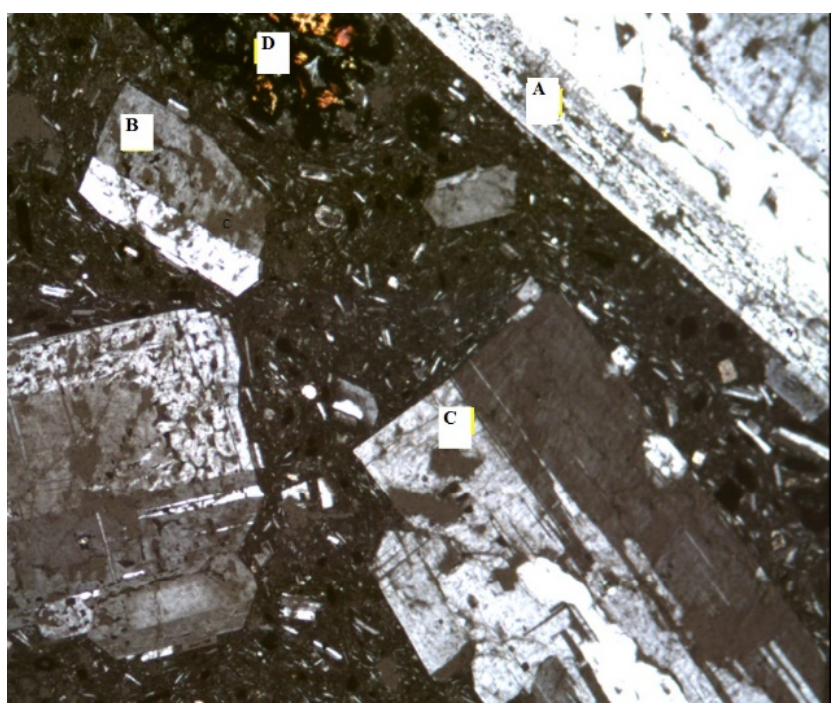

Figura 6. Sección delgada de un agregado andesítico, donde se muestran los cristales de plagioclasa en una pasta microlítica compuesta de feldespato. La microfotografía fue tomada a polar cruzada $4 \mathrm{X}$. 


\subsection{Análisis microestructural}

Por su parte en la Figura 7 se muestran las microfotografías de materias primas utilizadas en esta investigación, en la figura 7a se observa la morfología de que las diatomitas naturales donde se pueden apreciar algunas diatomeas, en la figura $7 \mathrm{~b}$ se presenta la diatomita calcinada en el cual se aprecia una cambio en la morfología con un incremento en el tamaño de poro y siendo más amorfa, por su parte en la figura $7 \mathrm{c}$ se observa la micrografía de los agregados reciclados, en donde se aprecian algunos feldespatos y silicatos, con presencia de calcita cubriendo algunas partículas, y en la figura $7 \mathrm{~d}$ se aprecian partículas de pumicita con bordes afilados, lo que es representativo de silicatos y poros característicos de las espumas volcánicas.
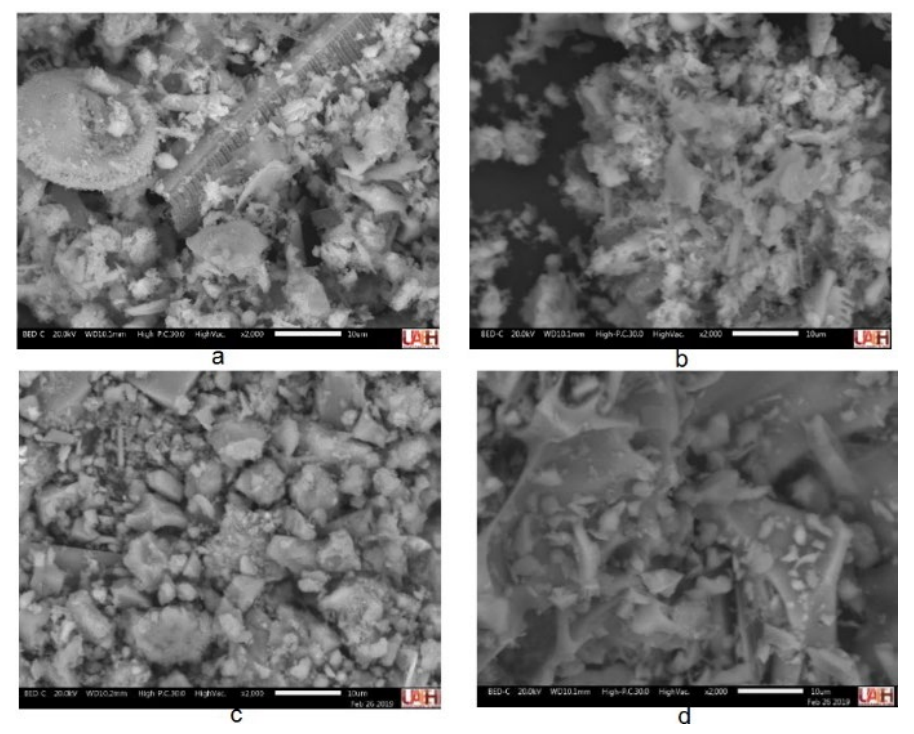

Figura 7. Morfología de las materias primas a) Diatomita natural, b) Diatomita calcinada, c) Agregados reciclados d) Pumicita

\subsection{Elaboración de bloques con agregados reciclados, pumicita y diatomita}

En la Tabla 3 se presenta la relación de las mezclas utilizadas para la fabricación de cada compuesto cerámico. Las cantidades de materiales se encuentran expresados en gramos y las medidas de cada compuesto cerámico será de $6 \times 10 \times 20 \mathrm{~cm}$ dando un volumen final de $1,200 \mathrm{~cm}^{3}$. Realizándose por quintuplicado en cada porcentaje de diatomita según la nmx-c-441-onncce-2013 para piezas no estructurales.
Tabla 3. Relación de las mezclas utilizadas para la fabricación de cada compuesto cerámico.

\begin{tabular}{cccccc}
\hline $\begin{array}{c}\text { Tipos de } \\
\text { diatomita }\end{array}$ & $\begin{array}{c}\text { Reemplazo } \\
\text { Diatomita } \\
(\%)\end{array}$ & $\begin{array}{c}\text { Diatomita } \\
(\mathrm{g})\end{array}$ & $\begin{array}{c}\text { Cemento } \\
(\mathrm{g})\end{array}$ & $\begin{array}{c}\text { Pumicita } \\
(\mathrm{g})\end{array}$ & $\begin{array}{c}\text { Agua } \\
(\mathrm{g})\end{array}$ \\
\hline \multirow{2}{*}{ DCF } & 5 & 5.4 & 102 & & \\
& 10 & 10.8 & 96.5 & 964.3 & 116 \\
& 15 & 16.2 & 91 & & \\
DC & 5 & 5.4 & 102 & & \\
& 10 & 10.8 & 96.5 & 964.3 & 116 \\
& 15 & 16.2 & 91 & & \\
DN & 5 & 5.4 & 102 & & \\
& 10 & 10.8 & 96.5 & 964.3 & 116 \\
\hline
\end{tabular}

\subsection{Resistencia a la compresión}

La influencia de los tipos de diatomitas en la resistencia del cemento Portland se aprecia en el gráfico de la Figura 8 donde se observa la resistencia a la compresión de la muestra del bloque con pumicita, cemento Portland y diatomita a la temperatura de curado de $20^{\circ} \mathrm{C}$ a $\operatorname{los} 7,14$ y 28 días.

Con base en esta prueba la resistencia de la muestra de block con diferentes contenidos de diatomita fue ligeramente inferior en la Diatomita Natural a la de la muestra de Referencia; a la edad de 7 días, las resistencias de la muestra de block de pumicita mezclado con la cantidad de mezcla de Diatomita Calcinada (DC) y Diatomita Calcinada con Fundente (DCF) fueron en su mayoría más altas que la de la muestra de Referencia (R).

En promedio las resistencias con la cantidad de mezcla del $5 \%$ en sustitución con el cemento de DC y DCF son $22 \mathrm{~kg} / \mathrm{cm}^{2}$ y 24.4 $\mathrm{kg} / \mathrm{cm}^{2}$ respectivamente siendo superiores a las de las muestras con la cantidad de mezcla del $10 \%$ y el $15 \%$. Se comparan con la resistencia del block normal $\left(20 \mathrm{~kg} / \mathrm{cm}^{2}\right)$ contra las muestras de DC y DCF, aumentando entre un $10 \%$ y $22 \%$ respectivamente. Dando como resultado que la Diatomita Calcinada con Fundente mezclada con el cemento a un 5\% puede mejorar la resistencia del espécimen de block de pumicita a temprana edad ( 7 días) en un $22 \%$ con respecto al block convencional.

El Ca $(\mathrm{OH})_{2}$ (Hidróxido de Calcio) se produjo por la hidratación del $\mathrm{SiO}_{2}$ (dióxido de Silicio) y las partículas de cemento en las partículas de diatomita y el gel de C-S-H (Silicatos de Calcio Hidratados) generado por $\mathrm{SiO}_{2}$ en la reacción de hidratación secundaria para aumentar la resistencia a la compresión del cemento (Liu et al, 2016). 

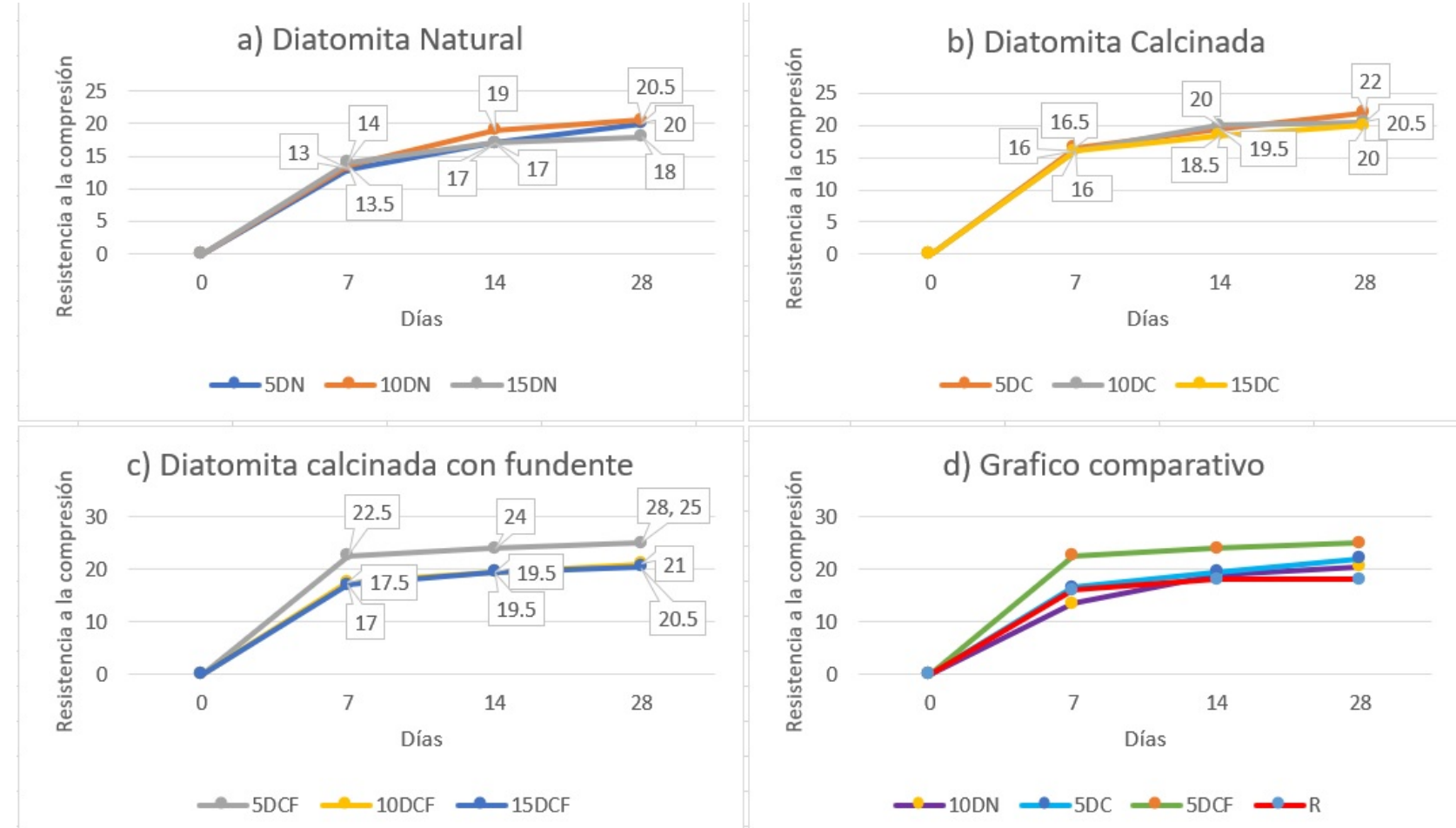

Figura 8. La resistencia a la compresión del bloque de piedra pómez mezclado con diferentes tipos de diatomita a) La muestra mezclada con diatomita normal ND b) La muestra mezclada con diatomita calcinada CD c) La muestra mezclada con diatomita calcinada con flujo DCF d) Comparación de los resultados óptimos obtenidos.

Posteriormente la influencia del Agregado reciclado en la resistencia del bloque de pumicita se aprecia en el gráfico de la Figura 9 a la temperatura de curado de $20^{\circ} \mathrm{C}$ a los 7,14 y 28 días. De igual manera se aprecia que los bloques de pumicita con agregados reciclados $10 \mathrm{AR}$ y $20 \mathrm{AR}$ son mayores, $21.6 \mathrm{~kg} / \mathrm{cm}^{2}$ y $22.2 \mathrm{~kg} / \mathrm{cm}^{2}$ respectivamente, que las del bloque de referencia (20 $\mathrm{kg} / \mathrm{cm}^{2}$ ), el que contiene el $30 \%$ de agregados reciclados en sustitución de la pumicita resultó menos eficiente $\left(18.5 \mathrm{~kg} / \mathrm{cm}^{2}\right)$.

Con base en los resultados se elige el bloque 20AR cuya resistencia aumentó en un $12 \%$ con respecto al bloque de referencia. Es importante mencionar que la resistencia alcanzada es ligeramente menor que la obtenida anteriormente solo con la sustitución de la diatomita calcinada con fundente ( $10 \%$ menor).

La calcita $\left(\mathrm{CaCO}_{3}\right)$ aparece en proporciones menores en todos los agregados reciclados. Por lo tanto, la calcita se debe predominantemente a la carbonatación de los productos de la hidratación del cemento en la pasta adherida a los agregados reciclados, como la portlandita $\mathrm{Ca}(\mathrm{OH})_{2}$ y los silicatos de calcio hidratado C-S-H $\left(\mathrm{CaO}-\mathrm{SiO}_{2}-\mathrm{H}_{2} \mathrm{O}\right)$. El carbonato de calcio se considera un cementante débil (Moreno et al, 2018), por eso disminuye su resistencia. Estas fases están carbonatadas como se describe en las Reacciones (1) y (2), (Shi et al, 2016).

$$
\begin{gathered}
\mathrm{Ca}(\mathrm{OH})_{2}+\mathrm{CO}_{2} \rightarrow \mathrm{CaCO}_{3}+\mathrm{H}_{2} \mathrm{O}-\cdots---(1) \\
\mathrm{C}-\mathrm{S}-\mathrm{H}+\mathrm{CO}_{2} \rightarrow \mathrm{CaCO}_{3}+\mathrm{SiO}_{2} \cdot \mathrm{nH}_{2} \mathrm{O}-\cdots-(2)
\end{gathered}
$$

Así mismo se determinó que el bloque elaborado con un $20 \%$ de agregado reciclado es el óptimo debido a que presenta una resistencia a la compresión de $22.2 \mathrm{~kg} / \mathrm{cm}^{2}$ mayor al de block de referencia $20 \mathrm{~kg} / \mathrm{cm}^{2}$ y ligeramente superior al de los otros bloques con el $10 \%$ y $30 \%$ de agregado reciclado $\left(21.6\right.$ y $18.5 \mathrm{~kg} / \mathrm{cm}^{2}$ respectivamente).

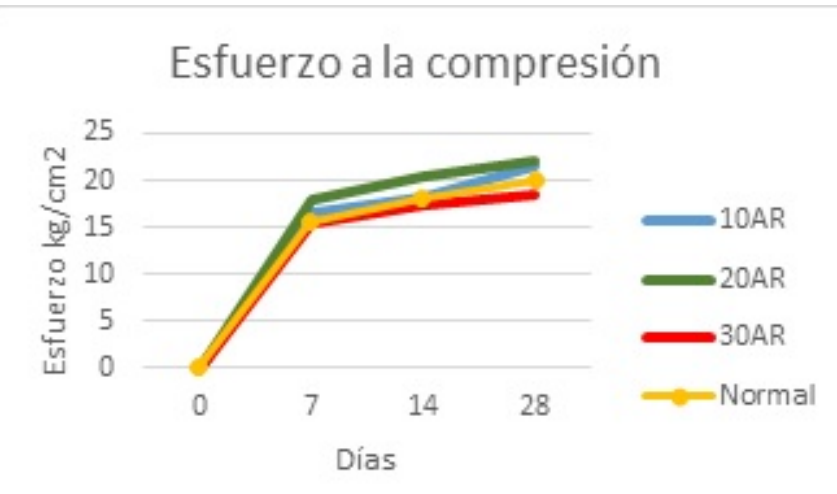

Figura 9. Gráfico que muestra la resistencia a la compresión de las muestras de bloques con áridos reciclados a los 7, 14 y 28 días.

En la Figura 10 a) se presentan las microfotografías donde se puede observar la fracción de arena de cuarzo y la porosidad esférica producto de aditivos aireantes junto con la cantidad de C$\mathrm{S}-\mathrm{H}$ que es mayor en el bloque común o comercial, mientras que en la figura 10b) se muestra la morfología en un bloque con diatomita al 5\% y Agregados Reciclados al 20\% en donde se encuentren las formaciones de silicatos cálcicos hidratados C-S-H (Tobermorita) cuya longitud es de aproximadamente 10 micras y su forma de aguja está definida, cuya función es la de darle resistencia al cemento, lo que conlleva a coadyuvar el aumento de la resistencia del bloque y respaldan los resultados obtenidos en las pruebas de resistencia mecánica. 


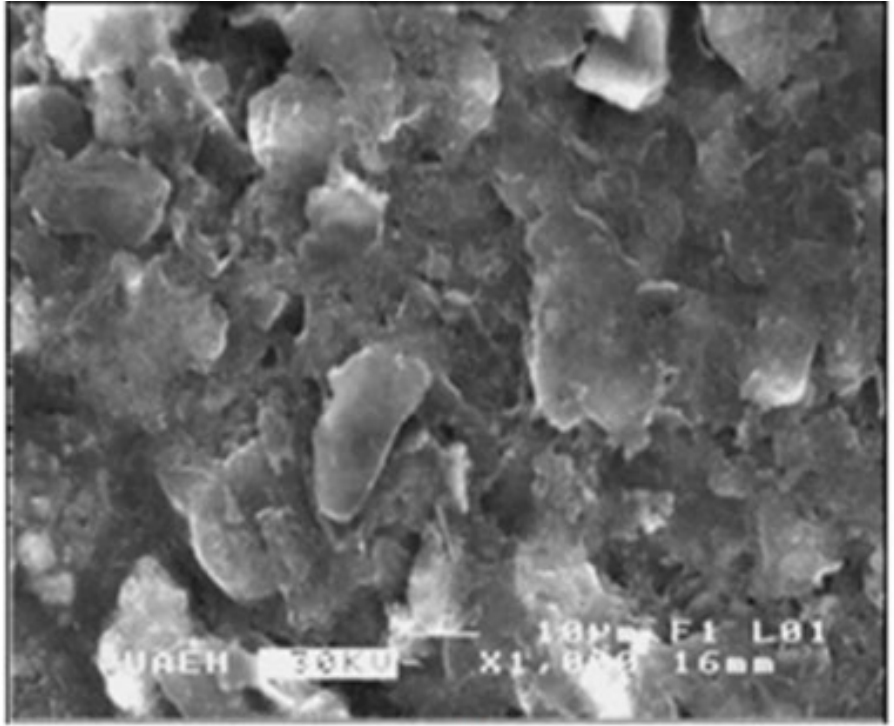

a

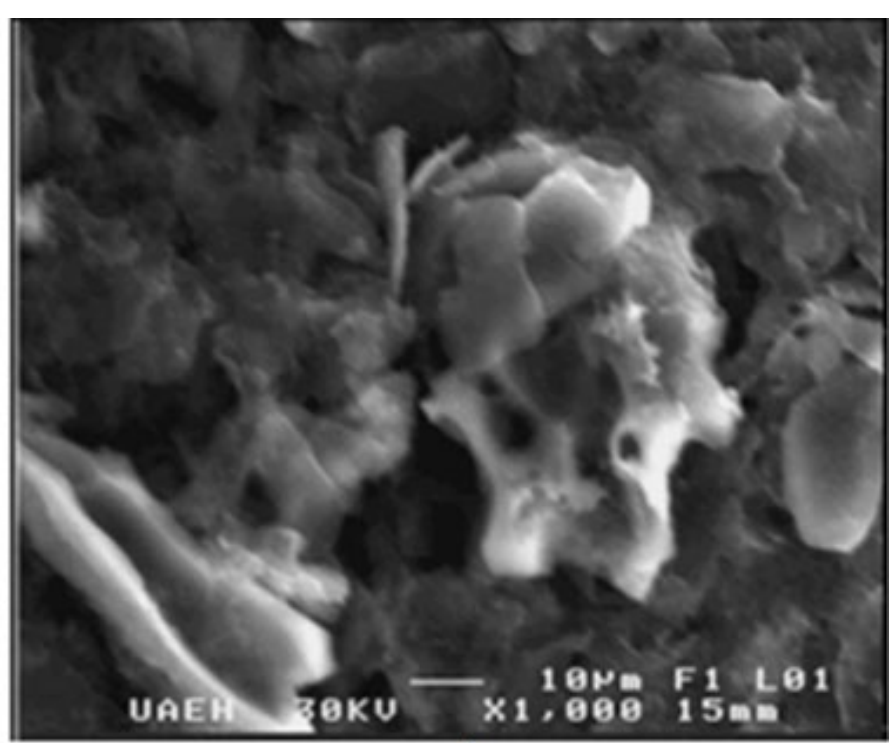

$\mathrm{b}$

Figura 10. Micrografías de bloques elaborados a) bloque comercial, b) bloque con diatomita al 5\% y Agregados Reciclados al $20 \%$.

\section{Discusión}

La industria del cemento contribuye con alrededor del 7\% del total de las emisiones mundiales de $\mathrm{CO}_{2}$ (aproximadamente $1.8 \mathrm{Gt}$ de emisiones de $\mathrm{CO}_{2}$ al año). (Barker et al, 2009), por lo anterior la sustitución de diatomita por cemento en la actualidad contribuye a la disminución de la huella de carbono generada por elaboración del cemento portland compuesto, lo cual ha favorecido al medio ambiente, ya que para la producción de cada tonelada de este produce en promedio 0,8 toneladas de $\mathrm{CO}_{2}$; por lo anterior Según la revista Civilizate por cada tonelada de cemento (20 bultos) utilizada en la elaboración de blocks de pumicita, se estaría ahorrando $50 \mathrm{~kg}$ de cemento ( 1 bulto) con el uso de la diatomita, con lo que se estaría reduciendo $40 \mathrm{~kg} \mathrm{de} \mathrm{CO}_{2}$ por tonelada de cemento producida, Por lo tanto, se reduciría unas $.09 \mathrm{Gt}$ de emisiones de $\mathrm{CO}_{2}$ al año (Barker et al, 2009), (Perera \& Dionisio, 2016). Pero al aumentar la cantidad de diatomita se producen más partículas de ésta que al no reaccionar sólo perjudican la resistencia de la pasta de cemento (Liu et al, 2016).

\section{Conclusiones}

En la elaboración de bloques de pumicita, se observó un incremento en la resistencia a la compresión a temprana edad (7 días) con la sustitución del $5 \%$ de diatomita calcinada con fundente (DCF). Incrementando un $22 \%$ a una edad de 28 días, con una resistencia cercana al $99 \%$. Notándose que a una edad de 7 días presenta un aumento del $20 \%$ comparado a un bloque de pumicita convencional. Esto ocurre debido a que el $\mathrm{Ca}(\mathrm{OH})_{2}$ (Hidróxido de Calcio) se produjo por la hidratación del $\mathrm{SiO}_{2}$ (dióxido de Silicio) y las partículas de cemento en las partículas de diatomita y el gel de CSH (Silicatos de Calcio Hidratados) generado por $\mathrm{SiO}_{2}$ en la reacción de hidratación secundaria para aumentar la resistencia a la compresión del cemento. por lo cual se recomienda dejar fraguar hasta los 28 días. La dosificación de los bloques de pumicita con la sustitución del agregado reciclado por la pumicita, obteniendo un ligero aumento de peso de $45 \mathrm{~g}$ en el bloque con $20 \%$ de Agregado Reciclado y un aumento de la cantidad de agua de un 7\%, siendo este las mejores condiciones obtenidas. Si bien el aumento con el árido reciclado fue menor que el obtenido sólo con la diatomita, sigue siendo mayor en comparación con el bloque de pumicita convencional. Haciendo énfasis en la parte sustentable del estudio, ya que se está reduciendo el impacto ambiental generado por los Residuos de Construcción y Demolición, proporcionalmente a la disminución de la pumicita al incorporarlo al block.

En base a datos obtenidos por diversas casas de materiales, con un bulto de cemento se pueden fabricar de 30 a 35 bloques de pumicita y en base al uso del $20 \%$ de agregados reciclados en sustitución con la pumicita, se estarían elaborando de 7 a 8 bloques más por cada bulto de cemento.

\section{Referencias}

D Oh, D.-Y., Noguchi, T., Kitagaki, R., Park, W.-J., 2014. CO2 emission reduction by reuse of 14 building material waste in the Japanese cement industry. Renew. Sustain. Energy Rev. 15 38, 796-810. https://doi.org/10.1016/j.rser.2014.07.036

Akhtar, A., \& Sarmah, A. (2018, March 7). Construction and demolition waste generation and properties of recycled aggregate concrete: A global perspective. Journal of Cleaner Production, 10.1016, p.6.

Aydin AC, Gül R. Influence of volcanic originated natural materials as additive on the setting time and some mechanical properties of concrete. Construction and building materials 2007; 21:1277-81.

Barker, D. J., Turner, S. A., Napier-Moore, P. A., Clark, M., \& Davison, J. E. (2009). CO2 capture in the cement industry. Energy procedia, 1(1), 8794.

Borgel I. (2007). Caracterización del yacimiento de diatomita de Loma Larga, Municipio de Acatlán, Hidalgo y evaluación de sus aplicaciones alternas. Tesis de Licenciatura, Área Académica de Materiales y Metalurgia, Universidad Autónoma del Estrado de Hidalgo.

Cáceres Sánchez, A. D. (2015). Estudio del hormigón simple elaborado con ladrillo reciclado y su incidencia en el peso específico y resistencia a compresión (Bachelor's thesis, Universidad Técnica de Ambato. Facultad de Ingeniería Civil y Mecánica. Carrera de Ingeniería Civil).

Cardoso, R.; Vasco Silva, R.; de Brito, J.; Dhir, R. Use of recycled aggregates from construction and demolition waste in geotechnical applications: A literature review. Waste Manag. 2016, 49, 131-145. [CrossRef] [PubMed] Hernández-Ávila, J., Salinas-Rodríguez, E., Cerecedo-Sáenz, E., ReyesValderrama, M., Arenas-Flores, A., Román-Gutiérrez, A. D., \& 
Rodríguez-Lugo, V. (2017). Diatoms and their capability for heavy metal removal by cationic exchange. Metals, 7(5), 169.

Kastis D. Properties and hydration of blended cements with calcareous diatomite. Cement Concrete Research 2006; 36:1821-6.

Liu J., Shao P., Wang S. (2016). The Influence of Diatomite on the Strength and Microstructure of Portland Cement. MATEC Web of Conferences, 67, 07-17.

LS-602, (2001), Method of Test for Sieve Analysis of Aggregates. Ministry of Transportation, Ontario, Laboratory Testing Manual. Available online: www.roadauthority.com (accessed on 6 May 2017).

Mete Z. Benefication of Kütahya-Alayunt Diatomite Ores (in Turkish). Akdeniz University Engineering Faculty Journal, Mining Section 1988;1:184-201.

Moreno, E., Hernández, J., Rangel, Y., Cerecedo, E., Arenas, A., Reyes, M., \& Salinas, E. (2018, mayo 21). Chemical and mineralogical characterization of recycled aggregates from construction and demolition waste from Mexico City. Minerals, 8, p. 237.

NMX-C-441-ONNCCE-2013. Industria de la construcción - mampostería, bloques tabiques o ladrillos y tabicones para uso no estructuralEspecificaciones y métodos de ensayo. Available online: https://www.onncce.org.mx/es/

Norma Mexicana NMX-C-036-ONNCCE-2013. Industria de la Construcción - Mampostería - Resistencia a la compresión de bloques, tabiques o ladrillos y tabicones y adoquines - Método de Ensayo. Available online: https://www.onncce.org.mx/es/venta-normas/fichastecnicas?view=item\&id=1730 (accessed on 20 February 2019).
Norma Mexicana NMX C-077-ONNCCE-1997. Agregados Para Concreto, Análisis Granulométrico, Métodos de Prueba. Organismo Nacional de Normalización y Certificación de la Construcción y Edificación, S.C. Available online: www.onncce.org.mx (accessed on 18 February 2017).

Norma Mexicana NMX C-111-ONNCCE-2017 (1) "Industria de la construcción - Agregados hidráulicos de concreto - Especificaciones y métodos de prueba" https://www.dof.gob.mx/nota detalle.php? codigo $=5543504 \&$ fecha $=12 / 1$ 1/2018, (accessed on 21 september 2018).

Omary, S., Ghorbel, E., \& Wardeh. (2016). Relationships between recycled concrete aggregates characteristics and recycled aggregates concretes properties. Constr. Build. Mater., 108, 163-174.

Perera, A. G., \& Dionisio, M. D. P. S. (2016). Alternativas de reducción de las emisiones de dióxido de carbono $\left(\mathrm{CO}_{2}\right)$ en la producción de cemento. Propuesta de un modelo de evaluación. Innovar: Revista de ciencias administrativas y sociales, 51-66.

Serralde, R., Sánchez, D., y López, J. (2015). Factibilidad de implementación de una empresa de base tecnológica para la fabricación de elementos urbanos, elaborados con base en residuos de la construcción y demolición (RCD): Analisis técnico de productos y maquinaria. Tesis de Licenciatura, Área Académica de Ingeniería, Universidad Autónoma del Estrado de Hidalgo.

Shi, C.; Li, Y.; Zhang, J.; Li, W.; Chong, L.; Xie, Z. Performance enhancement of recycled aggregate-Areview. J. Clean. Prod. 2016, 112, 466-472.

Stamatakis MG, Fragoulis D, Csirik G, Bedelean I, 2003. Pedersen S. The influence of biogenic micro-silica-rich rocks on the properties of blended cements. Cement Concrete Composites; 25:177-84. 